\title{
Research on Language Poverty Alleviation From the Perspective of Economics
}

\author{
Chengming $\mathrm{Ma}^{1, \mathrm{a}}$, Chen Lan ${ }^{1, \mathrm{~b}}$
}

\author{
${ }^{1}$ Northwest normal University, Gansu, Lanzhou, China 730070 \\ a3107000933@qq.com \\ b1093404970@qq.com
}

\begin{abstract}
In the course of our country's development, the problem of poverty has always been a very big problem, but in the view of economics, this study can better understand the influence of people's language on economy, and this paper mainly focuses on the influence of language diversity on economic growth and related factors, and the influence of language diversity on education and human capital accumulation, and also puts forward some strategies of language poverty alleviation.
\end{abstract}

Keywords: Economics; language poverty alleviation

\section{经济学视域下语言扶贫研究}

\author{
马成鸣 $1, \mathrm{a}$, 兰琛 $2, \mathrm{~b}$
}

\author{
1,2 西北师范大学，甘肃，兰州，中国 \\ $a, b 730070$ \\ 3107000933@qq.com \\ 1093404970@qq.com
}

\section{摘要}

在我国发展的过程中，贫困问题一直是一个非常大的问题，但是在经济学视域下，对此进行研究，那 么就能够更好的理解人们的语言对于经济的影响, 而本文就主要围绕语言多样性对经济增长及相关因 素的影响以及语言多样化对教育和人力资本积累的影响进行研究，同时也提出了一定的语言扶贫策略。 关键词:经济学; 语言扶贫

\section{1. 语言扶贫事业}

\section{1 语言扶贫}

语言扶贫主要是指提升语言文字的表达能力, 以不 同类型的语言形成因素和相关的语言政策结合在一起 进行语言扶贫, 并且语言扶贫是提高整体素质为主体的 活动。

语言扶贫的发展属于一种内在的发展, 国家对贫困 地区的居民通过制定相关语言文字的扶贫语言政策来 提高当地居民的语言文字表达能力的教育, 使当地贫困 居民能够有机会接触到我国传统的语言文字, 感受到语 言文字的鬼米力, 进而提升我国整体教育。

\section{2 语言精准扶贫}

我国对于扶贫工作的展开战略的重心就是为了能 够精准扶贫, 精准脱贫, 语言精准扶贫就是指我国要利 用相关政策，采取正确的扶贫方式针对扶贫对象进行精 准脱贫。

\section{3 普通话推广}

普通话作为我国语言当中的核心内容, 它具有较为 广泛的覆盖面, 并且国家对于普通话颁布的相关政策, 更加触动了普通话在我国的语言当中的发展，有效的提 升了普通话在我国人民生活当中的语言表达应用。然而 对于贫困居民的语言扶贫，不仅仅是为了让贫困居民能 够感受到语言文字的鬼米力，提升贫困居民的语言表达能 力，进而使更多的贫困居民可以讲述普通话，更是为了 
提高我国整体语言素质, 以及教育。然而对于用普通话 进行语言扶贫, 对我国经济发展具有一定的意义, 因为 普通话的推广能够使我国的文字更加规范, 也能够进一 步明确普通话在我国当中的法定地位, 并且我国相关教 育、广播影视等电视台都是采用普通话进行表述。因此, 普通话扶贫的同时也能够利用扶贫来推广普通话, 具有 非常远见的意义。

\section{2. 语言多样性对经济增长及相关因素的影响}

\section{1 语言多样性对经济增长的影响}

语言的多样性能够对经济产生非常大的影响, 而这 种影响是非常多面的, 同时这种影响的产生以及这种影 响的作用也是非常复杂的, 而且语言多样性往往会阻碍 到经济的发展, 而且其可能造成的影响具有非常强烈的 不确定性。以及语言多样性能够使交易成本大大提升, 并且不利于地区或者国家的经济发展, 但是这并不意味 着语言多样性没有任何积极作用, 在某种情况下, 语言 多样性也具有其独特的积极作用, 例如不同文化的交融 能够使得人力资本得到提升, 同时也能够使得产业和就 业率得到提升。而当产业和就业得到提升, 那么人力资 本就能够慢慢得到积累, 也就能够变相的推动经济的发 展。会阻碍经济增长的因素是非常难以解决的, 尤其是 方言的多样性, 语言多样性自然能够在一定程度上对经 济发展作出促进的作用, 而语言多样性不利于地区的经 济发展, 尤其是方言多样性。对于经济的发展而言, 具 备了非常显著的负面作用。如果受到语言多样性所带来 的影响, 那么经济的发展就很难得到提升。而之所以中 国地域经济差异非常大, 地理因素固然是一个非常关键 的因素, 但是方言差异也是其中非常重要的因素。

\section{2 语言多样性影响教育和人力资本积累}

会对经济增长造成阻碍的就是语言多样性, 而语言 多样性也会阻碍人力资本的积累, 如果能够消除方言的 多样性, 那么人均人力资本的积累都能够得以提升。而 且语言多样性对教育也有着非常大的影响, 并且是负面 影响。例如方言多样性会导致文盲率提升，而且会抑制 地区的人力资本提升。同时如果地区方言统一, 那么就 会对外来人口产生排斥心理, 而这样不利于地区经济的 发展。但是如果我国的教育水平能够得到不断的提升, 那么语言都能形成负面作用, 负面影响力会不断的削 弱。而这一点也说明教育可以在一定程度上缓解语言多 样性所带来的负面影响。那同时方言也会影响到人们的 就业情况, 对于部分劳动力而言, 很难跨区域进行工作。 而推广普通话教育，不仅仅是为了提高这部分人的区域 流动性, 也是为了能够推动地区的发展, 使劳动力市场 能够得到进一步的整合。

表 1 语言多样性对经济增长及相关因素的影响

\begin{tabular}{|c|c|}
\hline $\begin{array}{l}\text { 对于经济的发展而言, } \\
\text { 具备了非常显著的负 } \\
\text { 面作用。如果受到语言 } \\
\text { 多样性所带来的影响, } \\
\text { 那么经济的发展就很 } \\
\text { 难得到提升。而之所以 } \\
\text { 中国地域经济差异非 } \\
\text { 常大, 地理因素固然是 } \\
\text { 一个非常关键的因素, } \\
\text { 但是方言差异也是其 } \\
\text { 中非常重要的因素。 }\end{array}$ & $\begin{array}{l}\text { 例如方言多样性会导 } \\
\text { 致文盲率提升, 而县 } \\
\text { 抑制地区的人力资本 } \\
\text { 提升。同时如果地区方 } \\
\text { 言统一, 那么就会对外 } \\
\text { 来人口产生排斥心理, } \\
\text { 而这样不利于地区经 } \\
\text { 济的发展。但是如果我 } \\
\text { 国的教育水平能够得 } \\
\text { 到不断的提升, 那么语 } \\
\text { 言都能形成负面作用, } \\
\text { 负面影响力会不断的 } \\
\text { 削弱。而这一点也说明 } \\
\text { 教育可以在一定程度 } \\
\text { 上缓解语言多样性所 } \\
\text { 带来的负面影响。 }\end{array}$ \\
\hline
\end{tabular}

\section{3. 在经济学视域下的语言扶贫策略}

\section{1 开展语言扶贫需认识并遵循语言经济规 律}

虽然当前语言多样性会对语言能力以及经济发展 造成非常大的影响, 但是实际上语言会对这些事物所造 成的影响也具备一定的规律性, 如果能够正确的把握这 些规律，那么也能够找到正确的开展语言扶贫的途径。 而就现阶段而言, 所体现出的语言经济规律有以下几 种。一方面不同的语言对于不同的个体的影响也存在一 定的差异，而主要会受到不同个体的受教育程度，性别 因素以及自身收入等个性特征的差异的影响。另一方 面, 外语能力和普通话会对不同个体产生的影响也是存 在差异的, 缺乏普通话能力不利于国内人员的流动, 而 缺乏外语能力不利于国家与国家之间的人员流动。因此 在这一点中也能够看出, 语言扶贫时需要注意到对于不 同的个体而言有不同的需求，有不同的问题所在，所以 应该采取更加针对性的举措。同时语言会对经济发展所 造成的影响也会受到其他因素的影响, 因此如果其他因 素发生了变化, 那么语言对于经济发展所造成的影响也 会发生变化, 而这与大环境经济背景也有着非常大的关 系。

\section{2 开展语言扶贫需客观、全面地认识语言的 经济作用}

语言会影响到人力资本的积累, 同时也会影响到劳 动力的流动以及技术的扩散。因此也可以看出语言对经 济的影响的范围是非常大的, 而且语言对经济的影响会 直接深入到个体的收入情况, 而且具有传导的作用。而 语言对于经济的影响是非常隐性的, 往往不能马上就发 
现其造成的负面的作用，需要过了一定的周期才能够发 现其负面作用, 所以这也导致对于经济的影响往往会受 到忽略, 但是语言其实是一个重要的影响因素, 而且语 言会对经济造成的影响是伴随性的, 还需要结合其他的 因素, 才能够真正的显现出语言对经济的影响。例如语 言多样性并不是直接的影响人力资本的积累和劳动力 的流动, 主要是因为方言的多样性导致人们之间的交流 和沟通产生影响, 并且导致人们之间缺乏认同感。而因 为这些才渐渐的导致了劳动力流动和人力资本积累造 成影响。而这些因素本身就会影响到经济增长, 语言多 样性对于经济是存在一定的影响力的, 而同时人们的语 言能力也会对人们的收入情况造成非常大的影响。如果 人们不重视这种影响, 那么对于人们的个人发展也会有 非常大的负面作用, 而一种语言想要对经济起到促进的 作用, 那么就必须达到普及的程度, 只有具备一定规模 的语言统一才能够促进经济的发展。

\section{表 2 在经济学视域下的语言扶贫策略}

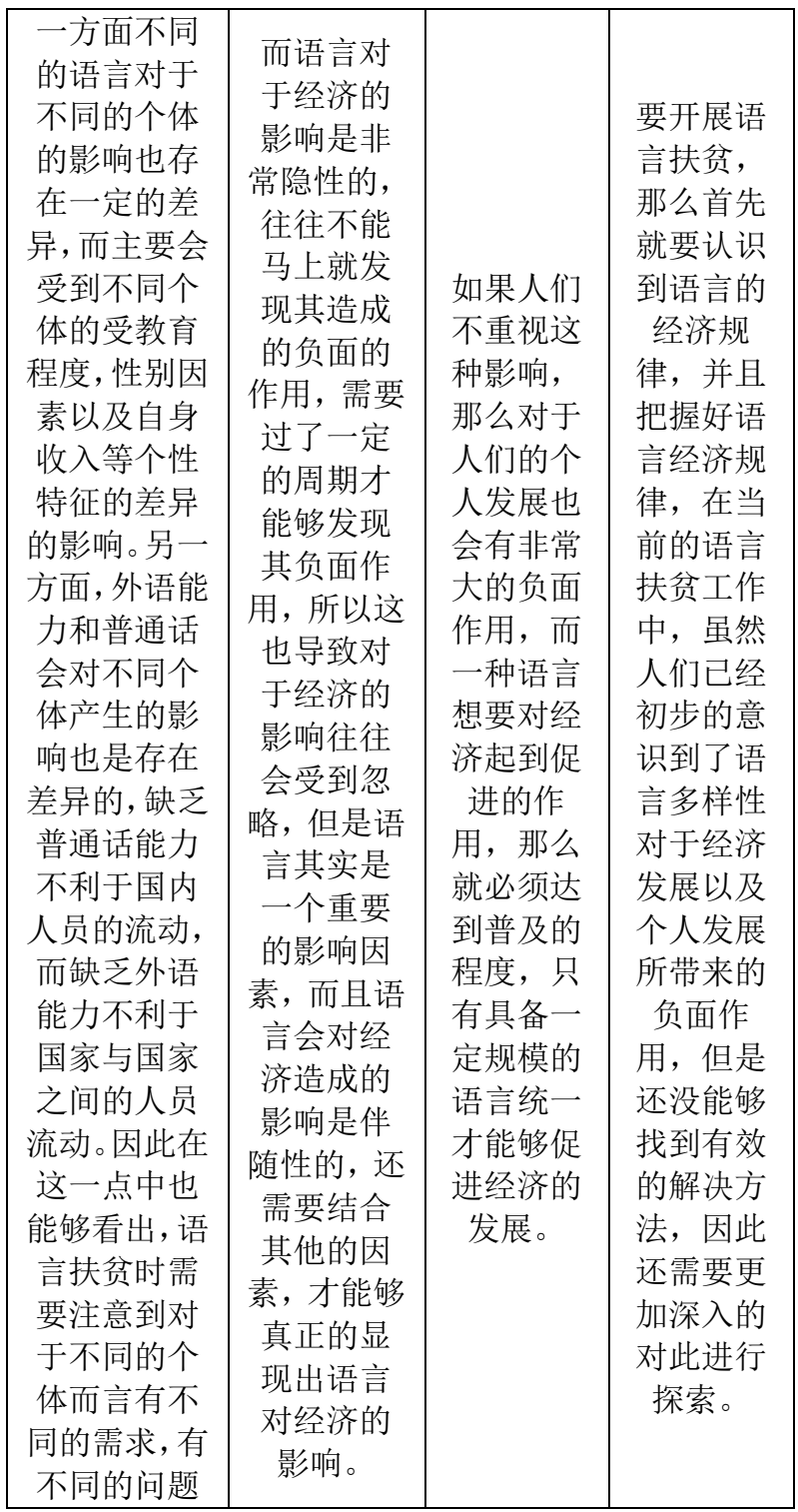

\begin{tabular}{|c|l|l|l|}
\hline $\begin{array}{c}\text { 所在, 所以应 } \\
\text { 该采取更加 } \\
\text { 针对性的举 } \\
\text { 措。 }\end{array}$ & & & \\
& & & \\
& & & \\
\hline
\end{tabular}

\section{4. 结语}

综上所述, 从古至今, 贫困一直是人类发展过程当 中的阶段性产物, 是所有国家都会面临的问题之一, 也 是一直以来人类共同面对和必须解决的重大问题。由于 贫困家庭受到经济状况影响, 对于人们的成长, 对事物 的认知以及心理健康都会产生一定的影响, 甚至对于人 们的语言表达能力也会产生影响, 进而也影响了我国整 体经济的发展。目前, 我国所实行的语言扶贫实践也正 是在为我国语言与贫困的研究提供了一次机遇。

因此, 要开展语言扶贫, 那么首先就要认识到语言 的经济规律, 并且把握好语言经济规律, 在当前的语言 扶贫工作中, 虽然人们已经初步的意识到了语言多样性 对于经济发展以及个人发展所带来的负面作用, 但是还 没能够找到有效的解决方法, 因此还需要更加深入的对 此进行探索。

\section{REFERENCES}

[1] Chunhui Wang. On the Role of Language Factors in Combating Poverty [J]. Jiang Han Academic Academic. 2018(05)

[2] Jifang Qiu, Niewei. Effect Mechanism of Dialect Skills on the Will of Urban Farmers —_ Based on the Dynamic Monitoring Data of the National Floating Population in 2014 [J]. Journal of Hunan Agricultural University (Social Sciences Edition). 2018(03)

[3] Gaojing, Linshu. Interprovincial boundaries, dialect boundaries and monovalent laws [J]. Financial research. 2018(04)

[4] Hailan Wan. Study on the Mechanism of Language Human Capital to Promote Economic Growth [J] Language Strategy Research. 2018(02)

[5] Giles Greene, Guohui Liu. On the Economic Value of Language and Its Diversity [J]. Journal of Yunnan Normal University (Philosophy and Social Sciences Edition). 2018(01)

[6] Hailan Wan. An Analysis of the Interaction Between Language Diversity and Economic Development [J]. Institutional Economics Research. 2017(04) 